\title{
Alleviation of Brain Edema and Restoration of Functional Independence by Bevacizumab in Brain-Metastatic Breast Cancer: A Case Report
}

\author{
Anna S. Berghoffa, Cornelia Sax ${ }^{a, g}$ Martin Klein ${ }^{b} \quad$ Julia Furtner ${ }^{c, g}$ \\ Karin Dieckmann ${ }^{\text {d, g }}$ Brigitte Gatterbauere,g Georg Widhalm ${ }^{\mathrm{e}, \mathrm{g}}$ \\ Margaretha Rudas ${ }^{f} \quad$ Christoph C. Zielinskia,g Rupert Bartsch ${ }^{\text {a, g }}$ Matthias Preusser ${ }^{a, g}$ \\ aDepartment of Medicine I, Medical University of Vienna, Austria; ${ }^{b}$ Department of Medical Psychology, VU University Medical Center, \\ Amsterdam, The Netherlands; 'Department of Neuroradiology; 'Department of Radiotherapy; ${ }^{\mathrm{e} D e p a r t m e n t}$ of Neurosurgery; \\ fDepartment of Pathology; ${ }^{\text {fC }}$ omprehensive Cancer Center, CNS Tumors Unit (CCC-CNS), Medical University of Vienna, Vienna, Austria
}

\section{Established Facts}

- Incidence of brain metastases (BM) in breast cancer is increasing, but treatment options are limited.

- In everyday practice, for local recurrence after local radiation treatment, a symptom-orientated treatment approach with corticosteroids for reduction of peritumoral edema is frequently chosen.

- Corticosteroid treatment has severe side effects.

\section{Novel Insights}

- A patient with brain-only metastatic breast cancer, after several local treatment approaches with surgery and radiotherapy, was successfully treated with bevacizumab monotherapy.

- The patient experienced significant alleviation of symptom burden, increased quality of life and improved neurocognitive functioning.

\section{Keywords}

Breast cancer - Brain metastases - Neurocognitive functioning - Bevacizumab - Symptom control . Brain edema

\section{Summary}

Background: Brain metastases (BM) are an increasing challenge in modern oncology, as treatment options especially after exhaustion of local treatment approaches are very limited. Patient and Methods: A long-term surviving patient with brain-only metastatic breast cancer, who presented at our department with massive corticosteroid-refractory brain edema with serious neuro- logical symptoms after exhaustion of all local therapy options, was started on bevacizumab. Results: Initiation of bevacizumab monotherapy led to rapid decrease of contrast-enhancing lesions and alleviation of brain edema, and allowed tapering and termination of corticosteroid administration. Neurological and neurocognitive function was restored and marked improvement in quality of life was observed. Conclusion: Our case highlights that bevacizumab may represent a feasible and effective salvage treatment option in selected patients with BM.

\section{KARGER}

Fax +497614520714

Information@Karger.com

www.karger.com

\section{(c) 2014 S. Karger GmbH, Freiburg}

$1661-3791 / 14 / 0092-0134 \$ 39.50 / 0$

Accessible online at:

www.karger.com/brc 


\section{Introduction}

Brain metastases (BM) are a frequent and life-limiting complication in cancer patients $[1,2]$. Treatment options include radiotherapy, stereotactic radiosurgery, neurosurgery, and best supportive care. Symptoms of increased cranial pressure and brain edema are treated most frequently with steroids. However, steroids can cause various serious side effects that further impair quality of life [3-7].

Here we report our encouraging experience with bevacizumab for control of steroid-refractory brain edema in a patient with brain-metastatic breast cancer. Bevacizumab is a humanized monoclonal antibody directed against vascular endothelial growth factor, thus inhibiting vascular proliferation, and is approved for various cancer entities [8]. In primary brain tumors bevacizumab was noted to be highly effective in reducing contrast-enhancement and peritumoral brain edema [9]. Unfortunately, only few systematic data are so far available on bevacizumab in patients with BM [10-12].

\section{Case Report}

A female patient was first diagnosed with breast cancer in March 2006 at the age of 43 years. An invasive, lobular breast cancer with expression of estrogen receptor (ER) and progesterone receptor (PR), a Ki67 index of $60 \%$, G3 grading, no HER 2 overexpression and a pathological staging of pT2pN0M0 was found. After breast-conserving surgery, the patient received external beam radiation therapy and iridium implants. The patient declined cytotoxic chemotherapy and received 2-year treatment with tamoxifen. In June 2008 the patient presented with neurological symptoms, and a solitary BM was detected in her left frontal lobe. CT scan revealed no extracranial metastatic disease. The BM diagnosisspecific graded prognostic assessment class was 2 (estimated median overall survival (OS) 15.1 months) [2]. The BM was resected neurosurgically, and histologically corresponded to a breast cancer BM with a triplenegative phenotype. In April 2009, a new BM located left centrally was treated by radiosurgery. In May 2010, the patient developed a BM left frontally and was treated with whole brain radiotherapy. In February 2011, the left frontal BM showed progression with extensive peritumoral edema, and was treated with stereotactic radiotherapy and a systemic therapy with capecitabine. In November 2011, progression of the left frontal BM was diagnosed and the patient was treated with liposomalencapsulated doxorubicin. In February 2012, a new BM was detected in the left operculum and was resected neurosurgically. In January 2013, the patient presented with massive neurological decline with paresis and prominent aphasia, rendering the patient wheelchair bound and with a Karnofsky performance score (KPS) of 40\%. Cranial magnetic resonance imaging (MRI) demonstrated a marked enlargement of the left frontal $\mathrm{BM}$ with prominent contrast enhancement and perifocal brain edema leading to compression of the left lateral ventricle (fig. 1A and C). Highdose corticosteroid therapy did not lead to significant neurological improvement, but the patient developed severe iatrogenic Cushing's syndrome. Owing to the extensive prior therapies, no more options for surgery or radiotherapy were seen. At this time, testing of neurocognitive functioning (NCF) using standardized psychometric instruments showed serious deficits regarding visual scanning speed, divided attention, verbal fluency, and to a lesser extent in the verbal memory domain [13]. Compared to a large sample of healthy controls [14-16] and using modified $t$-tests for single-case methodology with an alpha set at $\mathrm{p}<0.05[17,18]$,
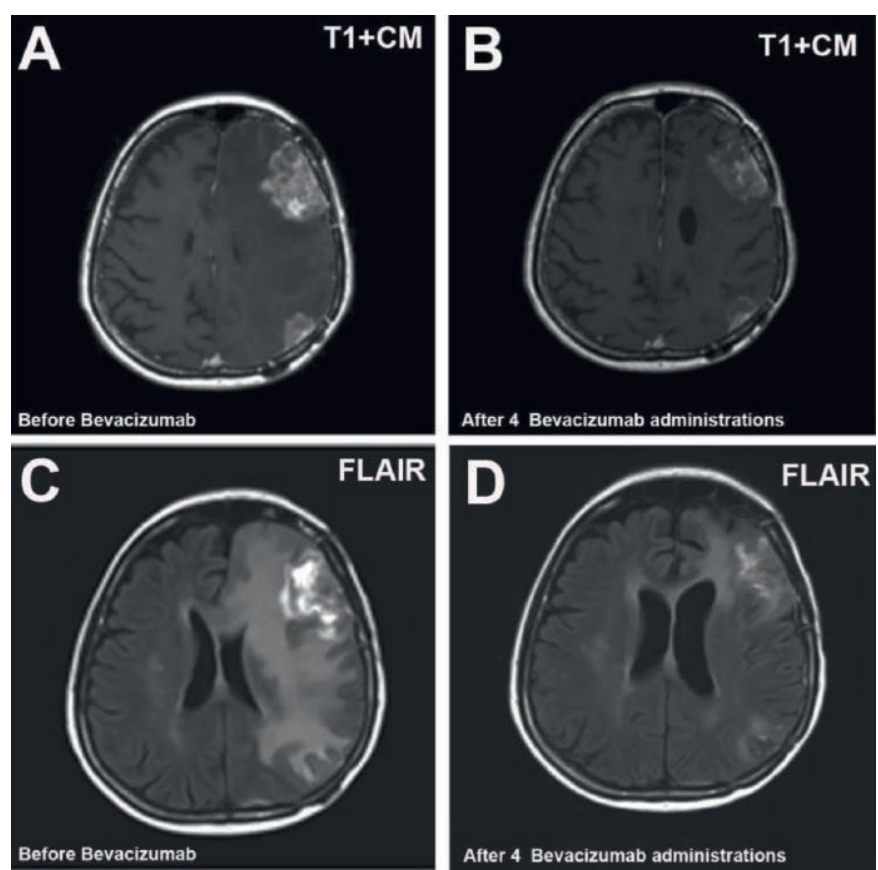

Fig. 1. Response to single-agent bevacizumab.

significant impairments of NCF were evident in our patient. Using established questionnaires (EORTC QLQ-BN20 and QLQ-C30) [19], the patient rated her health-related quality of life to be very poor.

We initiated bevacizumab therapy at a dose of $400 \mathrm{mg}$ intravenously every 2 weeks, a dosing schedule that is routinely used for recurrent glioblastoma at our institution and which has shown high efficacy against brain edema. Within a few days after initiation of bevacizumab, a marked improvement of clinical symptoms was evident and corticosteroid treatment was terminated. After 2 bevacizumab administrations, the patients' situation had dramatically improved. The patient was again fully ambulatory, able to care for herself, and started to walk her 2 dogs every day in the wood (KPS 90\%). Cranial MRI after a total of 4 bevacizumab applications showed an impressive reduction in both the size of contrastenhancing lesions and brain edema (fig. 1B and D). The patient's NCF improved markedly with only some minor residual limitations in divided attention capacity and recognition of previously presented auditory material. There was no longer a significant difference in NCF compared to healthy controls in most domains. Concerning quality of life, clinically relevant improvement was reported. At present our patient is alive and consistently well 64 months after diagnosis of BM.

\section{Discussion}

Previous reports have demonstrated that in up to $17 \%$ of breast cancer patients with BM, the CNS is the exclusive site of metastatic spread, and that long-term disease control and survival can be achieved by local interventions such as radiotherapy, stereotactic radiosurgery and neurosurgery [20]. After having undergone all local treatment options, our patient developed progressive contrast-enhancing BM with prominent perifocal brain edema and serious neurological symptoms. In this situation, best supportive care including high corticosteroid doses and involvement of hospice services 
is usually recommended. However, we decided to initiate bevacizumab monotherapy based on favorable experience with this drug in malignant primary brain tumors [21, 22]. Indeed, bevacizumab monotherapy led to a significant and clinically meaningful improvement, and allowed our patient to continue an independent and almost normal life.

Our case demonstrates that bevacizumab may represent a feasible and effective salvage treatment option in selected patients with BM, and this needs to be explored in further studies.

\section{Disclosure Statement}

The following authors declare financial assistance from Roche: Matthias Preusser (travel support and unrestricted research grants), Rupert Bartsch (travel support, speakers' honoraria and unrestricted research grants), Martin Klein (consultancy grants), and Christoph C. Zielinski (speakers' honoraria and advisory board allowance). All other authors declare no conflict of interest.

\section{References}

1 Davis FG, Dolecek TA, McCarthy BJ, Villano JL: Toward determining the lifetime occurrence of metastatic brain tumors estimated from 2007 United States cancer incidence data. Neuro Oncol 2012;14:1171-1177.

2 Sperduto PW, Kased N, Roberge D, et al.: Summary report on the graded prognostic assessment: An accurate and facile diagnosis-specific tool to estimate survival for patients with brain metastases. J Clin Oncol 2012;30:419-425.

$\checkmark 3$ Minisini AM, Moroso S, Gerratana L, et al.: Risk factors and survival outcomes in patients with brain metastases from breast cancer. Clin Exp Metastasis 2013;30:951-956.

4 Lin NU, Claus E, Sohl J, et al.: Sites of distant recurrence and clinical outcomes in patients with metastatic triple-negative breast cancer: High incidence of central nervous system metastases. Cancer 2008;113:2638-2645.

$\checkmark 5$ Berghoff A, Bago-Horvath Z, De Vries C, et al.: Brain metastases free survival differs between breast cancer subtypes. Br J Cancer 2012;106:440446.

6 Lin NU, Amiri-Kordestani L, Palmieri D, et al.: CNS metastases in breast cancer: Old challenge, new frontiers. Clinical Cancer Res 2013;19:64046418.

7 Lin NU, Winer EP: Brain metastases: The her2 paradigm. Clin Cancer Res 2007;13:1648-1655.
Braghiroli MI, Sabbaga J, Hoff PM: Bevacizumab: Overview of the literature. Expert Rev Anticancer Ther 2012;12:567-580.

$\checkmark 9$ Hattingen E, Jurcoane A, Daneshvar K, et al.: Quantitative 2 mapping of recurrent glioblastoma under bevacizumab improves monitoring for nonenhancing tumor progression and predicts overall survival. Neuro Oncol 2013;15:1395-1404.

10 Thompson EM, Frenkel EP, Neuwelt EA: The paradoxical effect of bevacizumab in the therapy of malignant gliomas. Neurology 2011;76:87-93.

11 Zustovich F, Ferro A, Lombardi G, et al.: Bevacizumab as front-line treatment of brain metastases from solid tumors: A case series. Anticancer Res 2013;33:4061-4065.

12 Boothe D, Young R, Yamada Y, et al.: Bevacizum$\mathrm{ab}$ as a treatment for radiation necrosis of brain metastases post stereotactic radiosurgery. Neuro Oncol 2013;15:1257-1263.

13 Meyers CA, Smith JA, Bezjak A, et al.: Neurocognitive function and progression in patients with brain metastases treated with whole-brain radiation and motexafin gadolinium: Results of a randomized phase III trial. J Clin Oncol 2004;22:157165.

14 Benedict RHB, Schretlen D, Groninger L, Brandt J: Hopkins verbal learning test - revised: Normative data and analysis of inter-form and test-retest reliability. Clin Neuropsychol 1998;12:43-55.
5 Ruff RM, Light RH, Parker SB, Levin HS: Benton controlled oral word association test: Reliability and updated norms. Arch Clin Neuropsychol 1996;11:329-338.

16 Tombaugh TN: Trail Making Test A and B: Normative data stratified by age and education. Arch Clin Neuropsychol 2004;19:203-214.

17 Crawford JR, Garthwaite PH: Investigation of the single case in neuropsychology: Confidence limits on the abnormality of test scores and test score differences. Neuropsychologia 2002;40:1196-1208.

18 Crawford JR, Howell DC: Comparing an individual's test score against norms derived from small samples. Clin Neuropsychol 1998;12:482-486.

19 Chiu L, Chiu N, Zeng L, et al.: Quality of life in patients with primary and metastatic brain cancer as reported in the literature using the EORTC QLQ-BN20 and QLQ-C30. Expert Rev Pharmacoecon Outcomes Res 2012;12:831-837.

20 Berghoff AS, Bago-Horvath Z, Ilhan-Mutlu A, et al.: Brain-only metastatic breast cancer is a distinct clinical entity characterised by favourable median overall survival time and a high rate of long-term survivors. Br J Cancer 2012;107:1454-1458.

21 Hofer S, Elandt K, Greil R, et al.: Clinical outcome with bevacizumab in patients with recurrent highgrade glioma treated outside clinical trials. Acta Oncol 2011;50:630-635.

22 Friedman HS, Prados MD, Wen PY, et al.: Bevacizumab alone and in combination with irinotecan in recurrent glioblastoma. J Clin Oncol 2009;27:47334740 . 